\title{
miR-133b reverses cisplatin resistance by targeting GSTP1 in cisplatin-resistant lung cancer cells
}

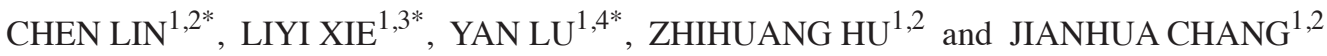 \\ ${ }^{1}$ Department of Oncology, Shanghai Medical College, Fudan University; Departments of ${ }^{2}$ Medical Oncology, \\ ${ }^{3}$ Radiation Oncology and ${ }^{4}$ Anesthesiology, Fudan University Shanghai Cancer Center, Shanghai 200032, P.R. China
}

Received January 18, 2017; Accepted January 8, 2018

DOI: $10.3892 /$ ijmm.2018.3382

\begin{abstract}
MicroRNAs play a critical role in chemoresistance and are implicated in various biological and pathological processes of cells. The objective of the present study was to explore the role of miR-133b and its mechanism in the regulation of cisplatin resistance and tumor progression in cisplatin-resistant non-small cell lung cancer (NSCLC) cells. Reverse transcription-quantitative polymerase chain reaction and western blot assays of the cisplatin-resistant cell lines A549/DPP and H1299/DDP displayed the reduced expression of miR-133b and increased expression of glutathione-S-transferase P1 (GSTP1) in the resistant cells compared with the respective parental cell lines A549 and H1299. Cell Counting kit-8, flow cytometry, colony formation and Transwell migration assays indicated that the overexpression of miR-133b increased the chemosensitivity to cisplatin and attenuated the proliferation and migration capacities of the cisplatin-resistant NSCLC cell lines in vitro. A dual-luciferase reporter assay demonstrated that miR-133b negatively regulated the expression of GSTP1 by targeting its 3'-untranslated region. In addition, the knockdown of GSTP1 by transfection with small interfering RNA exerted similar effects on cell chemosensitivity, proliferation and migration as did ectopic miR-133b expression, in addition to the upregulation of Bax and downregulation of $\mathrm{Bcl}-2$, survivin and matrix metalloproteinase expression. In conclusion, the present study findings provide
\end{abstract}

Correspondence to: Dr Jianhua Chang, Department of Medical Oncology, Fudan University Shanghai Cancer Center, 270 Dong'an Road, Shanghai 200032, P.R. China

E-mail: changjianhua@163.com

*Contributed equally

Abbreviations: miRNA, microRNA; NSCLC, non-small cell lung cancer; MMP, matrix metalloproteinase; 3'-UTR, 3'-untranslated region; mRNA, messenger RNA; GSTP1, glutathione-S-transferase P1; NC, negative control; siRNA, small interfering RNA; WT, wild type; HRP, horseradish peroxidase

Key words: miR-133b, cisplatin resistance, proliferation, migration, GSTP1, non-small cell lung cancer the insights that miR-133b reduces cisplatin resistance and its overexpression contributes to the suppression of the malignant growth and aggressiveness of cisplatin-resistant NSCLC cells by targeting GSTP1. This could potentially be exploited as a novel therapeutic strategy for the reversal of cisplatin resistance.

\section{Introduction}

Lung cancer is one of the most devastating malignancies as well as the leading cause of cancer-related morbidity and mortality globally (1). Non-small cell lung cancer (NSCLC) accounts for up to $85 \%$ of all lung cancers and has a 5-year survival rate of $\sim 15 \%$ (2). Despite multiple advances in therapeutic options over the years, at present, platinum-based chemotherapy remains the mainstay of adjuvant or first-line chemotherapy in NSCLC treatment $(3,4)$. Cisplatin is the most widely used platinum agent due to its therapeutic advantages. Compared with carboplatin-based chemotherapy, cisplatin-based chemotherapy is slightly superior in terms of response rate and in prolonging survival without being associated with an increase in severe toxic effects (5). Nevertheless, the clinical response in patients is frequently compromised by drug resistance, either intrinsic or acquired, resulting in chemotherapeutic failure and tumor relapse (6,7). Although researchers have delved into the modulation of cancer drug resistance at the molecular level, multiple mechanisms underlying cisplatin resistance require further clarification.

MicroRNAs (miRNAs) are endogenous single-stranded non-coding small RNAs 22 nucleotides in length, which regulate gene expression at the post-transcriptional level through binding to the 3' untranslated region (3'-UTR) of target messenger RNA (mRNA) (8). miRNAs often exhibit aberrant expression in human malignancies and function as either oncogenes or tumor suppressors depending on the biological roles of their target genes (9). Dysregulation of miRNA is involved in a wide range of biological processes, including cell proliferation, apoptosis and metastasis $(10,11)$. Recent studies have indicated that miRNAs play a role in chemoresistance to cancer treatment and are implicated in various biological and pathological processes of tumor cells $(12,13)$.

miRNA-133b has been indicated to be downregulated in several types of tumors, including gastric, prostate and colorectal cancers (14-16). Furthermore, the loss of miR-133b 
expression has been demonstrate to correlate with worse survival time in patients with lung cancer (17); however, to the best of our knowledge, there have not yet been any studies investigating its role in the regulation of the biological functions of drug-resistant lung cancer cells. In the present study, whether miR-133b modulates chemotherapy resistance, carcinogenesis and metastasis in cisplatin-resistant lung cancer cells was explored. The possible target for miR-133b was also predicted and verified in order to elucidate the effect of the interaction between this miRNA and its target gene on the biological activity of lung cancer cells.

\section{Materials and methods}

Cell culture. The human lung cancer cell lines A549 and H1299 were obtained from the Cell Bank of Type Culture Collection of Chinese Academy of Sciences (Shanghai, China). Cisplatin-resistant A549 (A549/DDP) cells were purchased from the Academy of Military Medical Sciences (Beijing, China), and cisplatin-resistant H1299 (H1299/DDP) cells were provided by the Institute of Lung Diseases, Xinqiao Hospital, Third Military Medical University (Chongqing, China). Cells were cultured in RPMI-1640 (Gibco; Thermo Fisher Scientific, Inc., Waltham, MA, USA) supplemented with $10 \%$ fetal bovine serum (Gibco; Thermo Fisher Scientific, Inc.) at $37^{\circ} \mathrm{C}$ in a humidified atmosphere with $5 \% \mathrm{CO}_{2}$. The culture medium for the A549/DDP and H1299/DDP cells included $1 \mu \mathrm{g} / \mathrm{ml}$ cisplatin to maintain the drug-resistant phenotype. Two days before each experiment, the culture medium was replaced by fresh medium without cisplatin to avoid the influence of the drug.

Drugs and antibodies. Cisplatin was purchased from Selleck Chemicals (Houston, TX, USA). Antibodies against glutathione-S-transferase P1 (GSTP1; cat. no. 3369; 1:1,000), survivin (cat. no. 2808; 1:1,000), B-cell lymphoma 2 (Bcl-2; cat. no. 15071; 1:1,000), Bcl-2-associated X protein (Bax; cat. no. 2774; 1:1,000), matrix metalloproteinase (MMP)-2 (cat.no.87809; 1:1,000),MMP-3 (cat.no. 14351; 1:1,000),MMP-9 (cat. no. 13667; 1:1,000) and $\beta$-actin (cat. no. 3700; 1:2,000) were purchased from Cell Signaling Technology, Inc. (Danvers, MA, USA). Horseradish peroxidase (HRP)-conjugated goat anti-rabbit and anti-mouse IgG (cat. nos. 111-035-003 and 115-035-003; each 1:10,000) antibodies were obtained from Jackson ImmunoResearch Laboratories, Inc. (West Grove, PA, USA).

Transient transfection. A549/DDP and H1299/DDP cells were seeded in 6-well plates. At $24 \mathrm{~h}$ after plating, cells were transfected with $100 \mathrm{nmol}$ miR-133b mimic or negative control (NC) mimic (Guangzhou RiboBio Co., Ltd., Guangzhou, China) using Lipofectamine 2000 (Invitrogen; Thermo Fisher Scientific, Inc., Waltham, MA, USA) according to the manufacturer's protocol. The effects were examined at $24 \mathrm{~h}$ post-transfection. The GSTP1 gene was knocked down using small interfering RNA (siRNA; Guangzhou RiboBio Co., Ltd.). The sequence of the GSTP1 siRNA was CCTACACCG TGGTCTATTT. The GSTP1-siRNA or control-siRNA (cat. no. siN05815122147-1-5) was diluted in Opti-MEM medium (Gibco, Thermo Fisher Scientific, Inc.) and transfected at a final concentration of $100 \mathrm{nM}$ using Lipofectamine 2000 in Opti-MEM medium in a 1:1 ratio. The mixture was incubated for $15 \mathrm{~min}$, then added to fresh culture medium and incubated with the cells for $48 \mathrm{~h}$. The transfected cells were then processed for subsequent experiments.

Reverse transcription-quantitative polymerase chain reaction (RT-qPCR) analysis. Total cellular RNA was extracted using TRIzol Reagent (Invitrogen; Thermo Fisher Scientific, Inc.). The RNA sample (500 ng) was then reverse transcribed into cDNA using a PrimeScript ${ }^{\mathrm{TM}}$ RT Reagent kit (Takara Bio, Inc., Otsu, Japan) with reaction at $37^{\circ} \mathrm{C}$ for $15 \mathrm{~min}$, followed by $85^{\circ} \mathrm{C}$ for $5 \mathrm{sec}$ and then cooling to $4^{\circ} \mathrm{C}$. For mRNA quantification, qPCR was performed using SYBR Premix Ex Taq (Takara Bio, Inc.) with the Applied Biosystems Prism 7900HT system (Applied Biosystems; Thermo Fisher Scientific, Inc.). The thermocycling conditions were 1 cycle of $95^{\circ} \mathrm{C}$ for $5 \mathrm{~min}$ and 40 cycles of $95^{\circ} \mathrm{C}$ for $5 \mathrm{sec}$ and $60^{\circ} \mathrm{C}$ for $34 \mathrm{sec}$. The GSTP1 primers were as follows: Forward, 5'-CCTGTACCAGTCCAATACCATCCT-3' and reverse, 5'-TCCTGCTGGTCCTTCCCATA-3'. Primers for miR-133b: RT, 5'-GTCGTATCCAGTGCAGGGTCCGAGGTA TTCGCACTGGATACGAC-3' (to replace the RT Primer mix of the PrimeScript RT reagent kit), forward, 5'-CTTTGGTCC CCTTCAACCA-3' and reverse, 5'-CTTTGGTCCCCTTCA ACCA-3'. Primers for U6: Forward, 5'-CTCGCTTCGGCA GCACA-3' and reverse, 5'-AACGCTTCACGAATTTGCGT-3'. Primers for $\beta$-actin: Forward, 5'-GCACCACACCTTCTACAA TGAGC-3' and reverse, 5'-GGATAGCACAGCCTGGATAGC AAC-3'. The levels of mature miRNA-133b expression were normalized to U6 and those of GSTP1 mRNA were normalized to $\beta$-actin mRNA. The fold change in expression was calculated using the $2^{-\triangle \Delta C q}$ method (18).

Dual-luciferase reporter assay. Using the web-based miRNA program TargetScan (http://www.targetscan.org/), it was predicted that hsa-miR-133b should bind with the 3'-UTR of GSTP1. The wild-type (WT) and mutant 3'-UTR of GSTP1 were independently cloned into a pGL3 luciferase vector (Promega Corporation, Madison, WI, USA) containing the Renilla luciferase gene. The WT sequence for the GSTP1 3'-UTR was GGGTTGGGGGGACTCTGAGCGGGAGGCAGAGTT TGCCTTCCTTTCTCCAGGACCAATAAAATTTCTAAGA GAGCTA, and the mutant sequence was GGGTTGGGGGG ACTCTGAGCGGGAGGCAGAGTTTGCCTTCCTTTCTCC ATACTAGCTAAA ATt TCTAAGAGAGCTA. For the luciferase assay, HEK 293T cells (Cell Bank of Type Culture Collection of Chinese Academy of Sciences, Shanghai, China) were cotransfected with the miR-133b mimic or NC mimic and the luciferase reporter plasmid using Lipofectamine 2000. At $48 \mathrm{~h}$ post transfection, firefly luciferase activity was measured using a Dual-Luciferase Reporter Assay System (Promega Corporation), according to the manufacturer's protocol. Firefly luciferase activity was normalized to Renilla luciferase activity for each well.

Colony formation assays. At $24 \mathrm{~h}$ after the transient transfection, 500 cells were re-seeded in 6-well plates in triplicate. Following 10 days of incubation, the colonies were fixed with $4 \%$ paraformaldehyde for $30 \mathrm{~min}$ and stained with $1 \%$ crystal violet for $2 \mathrm{~h}$ at room temperature. The plates were then 
washed and dried before photographic images were captured. The colony numbers were counted and the sizes of colonies were observed.

Cell viability assays. In the growth inhibition assay, transfected cells were seeded at a density of 8,000 cells/well in 96-well culture plates and incubated overnight. Following cell adhesion, cisplatin was applied at a series of concentrations $(1,2,4,8,16,32,64$ and $128 \mu \mathrm{M})$ and the cells were cultured for a further $48 \mathrm{~h}$. Cell survival was then assayed using a Cell Counting kit (CCK)-8 assay (Dojindo Molecular Technologies, Inc., Kumamoto, Japan). In the cell proliferation assay, transfected cells were seeded at a density of 5,000 cells/well, and CCK- 8 assays were performed to examine the cell viability following 24,48 and $72 \mathrm{~h}$ of culture.

Cell apoptosis analysis. Cells seeded in 6-well plates (1x105/well) were transfected with miR-133b mimic or NC mimic for $24 \mathrm{~h}$, and then treated with $10 \mu \mathrm{M}$ cisplatin for $48 \mathrm{~h}$. Cell apoptosis was assessed using flow cytometry with staining of the cells using an Annexin V/propidium iodide (PI) kit (cat. no. 556547; BD Biosciences, Franklin Lakes, NJ, USA). Briefly, cells were collected and washed twice in ice-cold PBS. The washed cells $\left(2 \times 10^{5}\right)$ were resuspended in $100 \mu \mathrm{l}$ binding buffer (included in the kit), and stained with $5 \mu \mathrm{l}$ Annexin V and $5 \mu \mathrm{l}$ PI. Following incubation for $15 \mathrm{~min}$ in the dark, flow cytometry was performed. A flow cytometer (Cytomics FC 500 MPL; Beckman Coulter, Inc., Brea, CA, USA) was utilized to evaluate the apoptotic levels in each sample following the manufacturer's protocol.

Cell migration assay. Migration experiments were carried out in a chamber with a BD Falcon ${ }^{\mathrm{TM}}$ Cell Culture insert (pore size, $8 \mu \mathrm{m}$; BD Biosciences). In these experiments, $2 \times 10^{4}$ cells in $200 \mu \mathrm{l}$ serum-free RPMI-1640 medium were added to the top chamber of the insert and $600 \mu 110 \%$ fetal bovine serum-containing medium (Gibco; Thermo Fisher Scientific, Inc.) was added to the lower chamber. Following $24 \mathrm{~h}$ incubation at $37^{\circ} \mathrm{C}$, the cells that had migrated to the lower chamber were fixed in $4 \%$ paraformaldehyde for $30 \mathrm{~min}$ and stained with $1 \%$ crystal violet for $2 \mathrm{~h}$ at room temperature. Stained cells were counted in five different fields in each well under an inverted microscope at a magnification of x200 (Olympus Corporation, Tokyo, Japan).

Western blot analysis. Total protein lysates were obtained from cultured cells with a mixture of radioimmunoprecipitation assay buffer (Beyotime Institute of Biotechnology, Shanghai, China), phosphatase inhibitor cocktail and protease inhibitor cocktail (both BioTool AG, Kirchberg, Switzerland). Protein concentrations were determined using a BCA protein assay kit (Beyotime Institute of Biotechnology). Cell extracts (20 $\mu \mathrm{g} /$ well) were separated by $10 \%$ sodium dodecyl sulfate polyacrylamide Tris- $\mathrm{HCl}$ gel electrophoresis and transferred onto polyvinylidene fluoride membranes. The membranes were then blocked with 5\% skimmed milk in Tris-buffered saline with Tween-20 (TBST) for $1 \mathrm{~h}$ at room temperature and probed with primary antibodies against GSTP1, survivin, Bcl-2, Bax, MMP-2, MMP-3, MMP-9 and $\beta$-actin overnight at $4^{\circ} \mathrm{C}$. After washing with TBST, the membranes were incubated with HRP-conjugated secondary antibody for $1 \mathrm{~h}$ at room temperature, then washed three times with TBST. The proteins were visualized with Pierce ${ }^{\mathrm{TM}}$ enhanced chemiluminescence reagents (Thermo Fisher Scientific, Inc.) and detected using a luminescent image analyzer (ImageQuant LAS4000 mini; GE Healthcare Life Sciences, Little Chalfont, UK).

Statistical analysis. Statistical analysis was performed using GraphPad Prism 5.0 software (GraphPad Software, Inc., La Jolla, CA, USA). Differences between two groups were analyzed using the Student's t-test, one-way analysis of variance was applied for the comparison of more than two groups, and post hoc tests were conducted using the Bonferroni correction. $\mathrm{P}<0.05$ was considered to indicate a statistically significant difference.

\section{Results}

Expression of miR-133b and GSTP1 in A549/DPP and H1299/DDP cells compared with their parental cell lines. Survival curves of the A549/DDP, A549, H1299/DDP and H1299 cells in response to various doses of cisplatin were constructed. CCK-8 growth inhibition assays demonstrated that the half maximal inhibitory concentration $\left(\mathrm{IC}_{50}\right)$ of cisplatin in the A549/DDP cells was $\sim 6$ fold higher than that in the A549 cells, and the H1299/DDP cells exhibited $~ 4$-fold greater resistance compared with the H1299 cells (Fig. 1A). The expression levels of miR-133b and GSTP1 protein in the parental and cisplatin-resistant cells were monitored using RT-qPCR and western blot analysis, respectively. As shown in Fig. 1B, miR-133b had 2.3- and 3.5-fold lower mean expression levels in the A549/DPP and H1299/DDP cells compared with the respective parental cells $(\mathrm{P}<0.05$; Fig. 1B). By contrast, the GSTP1 protein expression was upregulated in the two cisplatin-resistant cell lines compared with the parental cell lines (Fig. 1C).

Upregulation of miR-133b increases the cisplatin chemosensitivity of cisplatin-resistant NSCLC cells. To investigate whether miR-133b modulates the sensitivity of A549/DPP and H1299/DDP cells to cisplatin, the cells were transfected with miR-133b mimic or $\mathrm{NC}$ mimic. The $\mathrm{IC}_{50}$ of cisplatin in the A549/DDP cells was significantly decreased following transfection with miR-133b mimic compared with those in the blank and NC groups $(6.1 \pm 0.7$ vs. $20.7 \pm 1.5$ and $20.5 \pm 0.5 \mu \mathrm{M}$, respectively; both $\mathrm{P}<0.05$; Fig. 2A). Similarly, the miR-133b mimic sensitized H1299/DDP cells to cisplatin by $\sim 2.8$ fold compared with the blank and $\mathrm{NC}$ groups $\left(\mathrm{IC}_{50}\right.$ values $4.9 \pm 0.1$ vs. $14.2 \pm 0.5$ and $14.0 \pm 0.8 \mu \mathrm{M}$, respectively; both $\mathrm{P}<0.05)$, as evidenced by the growth inhibition curve (Fig. 2B). To further assess the role of miR-133b in the apoptosis of cisplatin-resistant cells when exposed to cisplatin treatment, flow cytometric assays were performed. As shown in Fig. 2C and D, compared with the NC and blank groups, the miR-133b mimic significantly promoted the cisplatin-induced apoptosis of A549/DDP and H1299/DDP cells. Annexin V/PI-based apoptosis analysis revealed that the transfection of A549/DDP and H1299/DDP cells with miRNA-133b mimic increased the percentage of apoptotic cells compared with those in the blank and NC groups $(17.9 \pm 0.8$ vs. $3.8 \pm 0.2$ vs 
A

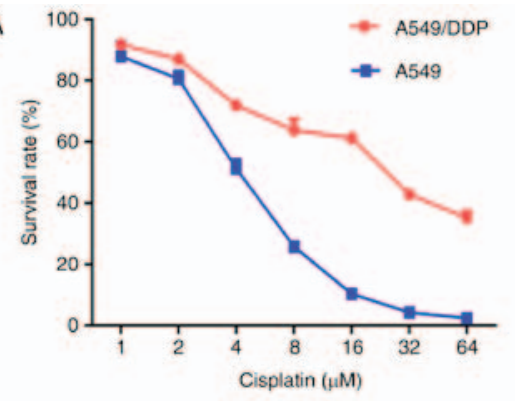

B

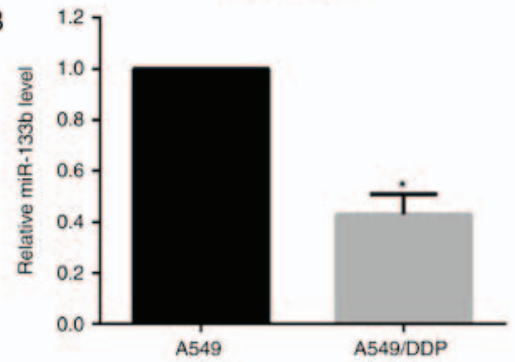

c

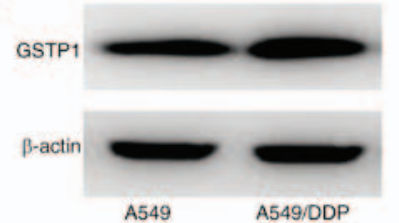

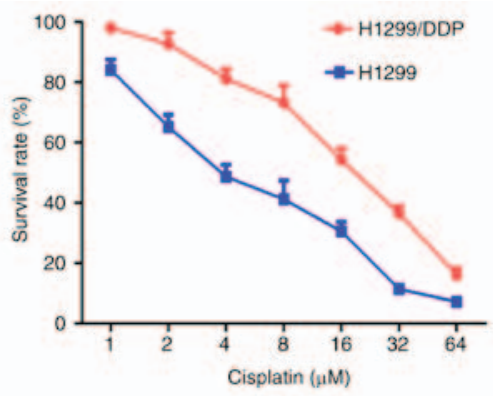
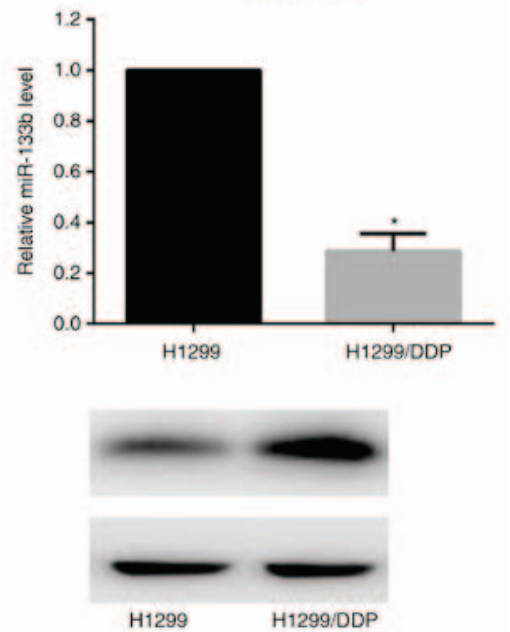

Figure 1. Survival rate and expression of miR-133b and GSTP1 in cisplatin-resistant NSCLC cells compared with the parental cell line. (A) Cell Counting kit-8 assays were used to determine the half maximal inhibitory concentration of cisplatin in A549/DDP, A549, H1299/DDP and H1299 cells. (B) Reverse transcription-quantitative polymerase chain reaction demonstrates lower expression levels of miR-133b in A549/DPP and H1299/DDP cells compared with the respective parental cells. (C) Western blot analysis reveals that the GSTP1 protein level is upregulated in A549/DPP and H1299/DDP cells compared with the respective parental cells. Data are the mean \pm standard deviation of three separate experiments. ${ }^{*} \mathrm{P}<0.05$ vs. the respective parental cell line. miR, microRNA; GSTP1, glutathione-S-transferase P1; NSCLC, non-small cell lung cancer; A549/DDP, cisplatin-resistant A549; H1299/DDP, cisplatin-resistant H1299.

$4.0 \pm 0.7 \%$, respectively, in the A549/DDP cells, and $15.3 \pm 0.7$ vs. $6.2 \pm 0.7$ vs. $6.6 \pm 1.0 \%$, respectively, in the H1299/DDP cells; all $\mathrm{P}<0.05)$. Western blotting demonstrated decreased expression levels of the anti-apoptotic proteins Bcl-2 and survivin, and concomitant activation of the pro-apoptotic protein Bax in the miR-133b mimic groups compared with the NC and blank groups in the two resistant cell lines (Fig. 2E). Therefore, these data suggest that miR-133b enhances the cisplatin chemosensitivity of cisplatin-resistant NSCLC cells via the promotion of apoptosis.

miR-133b inhibits the proliferation and clonogenesis of A549/DDP and H1299/DDP cells. To ascertain whether miR-133b affects the proliferative abilities of A549/DDP and H1299/DDP cells, CCK-8 assays were performed. Following transfection with miR-133b mimic, the proliferation of A549/DDP and H1299/DDP cells was significantly inhibited at days 2-4 ( $\mathrm{P}<0.05$; Fig. 3A). Consistent with this, colony formation assays demonstrated significantly lower colony-forming numbers of A549/DDP cells in the miR-133b mimic group compared with the blank and miR-NC groups $(71.3 \pm 8.4$ vs. $128.7 \pm 7.6$ and $120.3 \pm 4.2$, respectively; both $\mathrm{P}<0.05$; Fig. 3B). Similar results were confirmed in the H1299/DDP cell groups $(55.3 \pm 4.1$ vs. $96 \pm 6.5$ and $90.7 \pm 5.7$, respectively; both $\mathrm{P}<0.05$; Fig. $3 \mathrm{C}$ ). These results indicate that miR-133b impeded the proliferation of cisplatin-resistant NSCLC cells.
miR-133b attenuates the migration of A549/DDP and $H 1299 / D D P$ cells. To further explore the role of miR-133b in the migration of A549/DDP and H1299/DDP cells, Transwell chamber migration assays were conducted to assess the effect of miR-133b on the number of migrating cells. The migratory capacities of the A549/DDP cells were reduced by 2.7 fold by the overexpression of miR-133b compare/d with those of the blank and NC groups $(87.3 \pm 6.6$ vs. $238.3 \pm 13.9$ and $232.0 \pm 16.6$, respectively; both $\mathrm{P}<0.05$; Fig. 4A) and those of the H1299/DDP cells were reduced by $\sim 1.7$ fold $(251.3 \pm 18.0$ vs. $451.3 \pm 21.2$ and $438.0 \pm 22.8$, respectively; both $\mathrm{P}<0.05$; Fig. 4B). The expression of metastasis-associated proteins was then evaluated using western blotting. Consistent with the lower metastasis capacity following transfection with miR-133b mimic, the downregulation of MMP2, MMP3 and MMP9 was observed in the miR-133b-transfected cells compared with the blank and NC groups (Fig. 4C).

GSTP1 is a direct target of miR-133b. The identification of miRNA-regulated gene targets is essential to clarify the molecular mechanisms by which miRNAs mediate cancer progression. The target prediction program TargetScan Human 7.0 indicated that GSTP1 is a potential target gene of miR-133b (Fig. 5A). To confirm that miR-133b regulates GSTP1 expression by directly binding to the 3'-UTR of GSTP1, the WT or mutant GSTP1 3'-UTR was cloned into a vector downstream of the luciferase reporter gene and co-transfected with miR-133b 

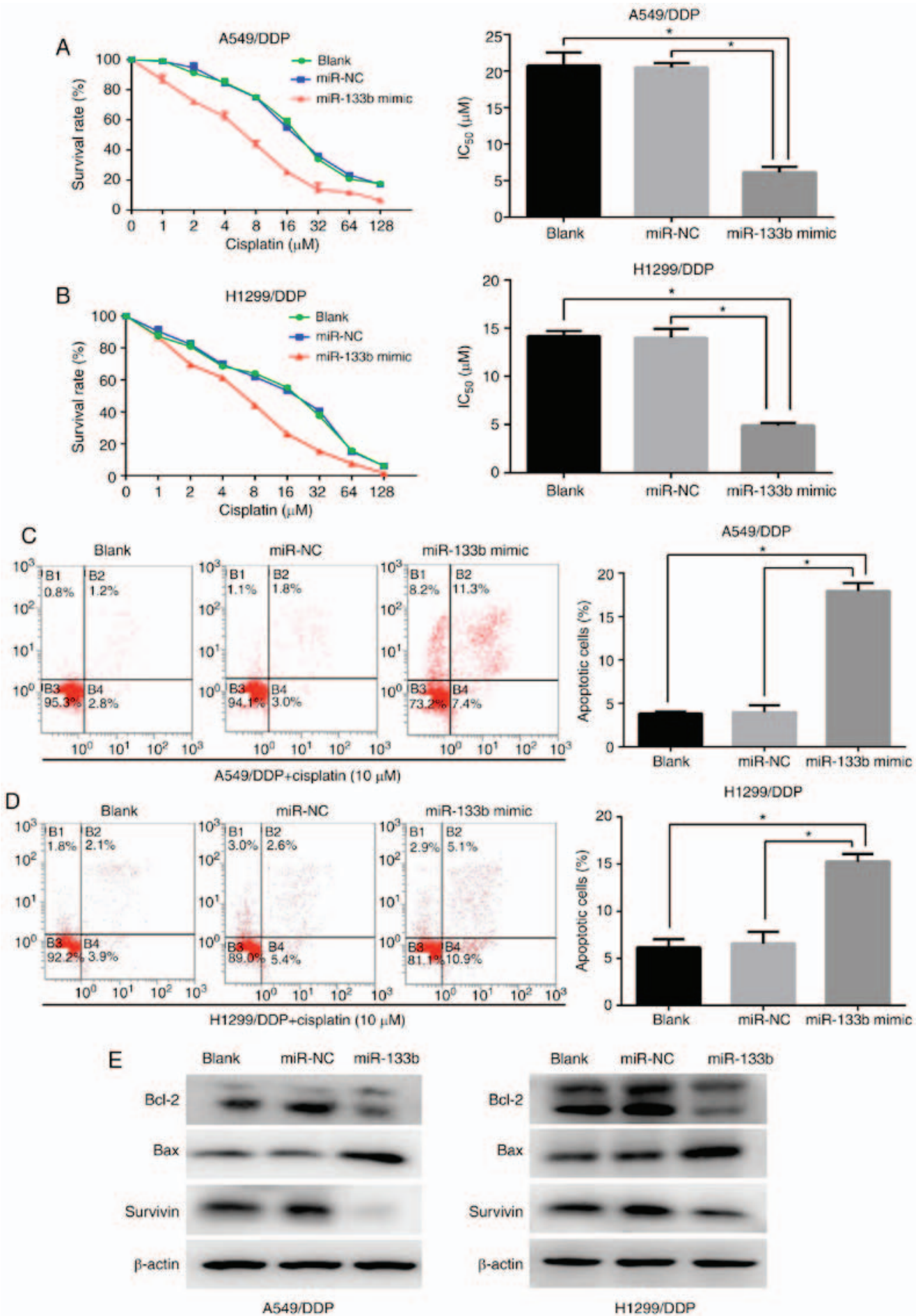

miR-133b mimic
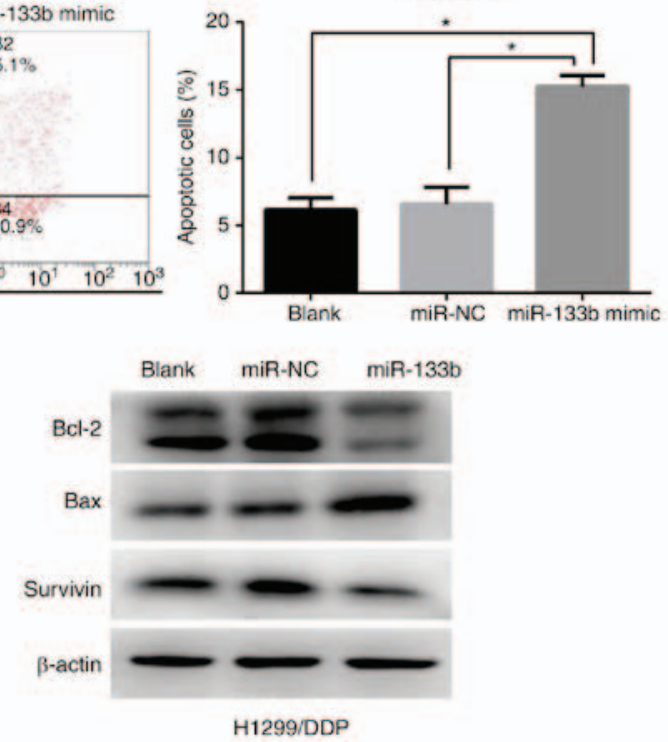

Figure 2. Upregulation of miR-133b increases the cisplatin chemosensitivity of cisplatin-resistant NSCLC cells. CCK-8 assays were used to determine the $\mathrm{IC}_{50}$ values of cisplatin following the transfection of (A) A549/DDP cells and (B) H1299/DDP cells with miR-133b mimic or negative control. Flow cytometric assays detected the cell apoptosis of (C) A549/DDP and (D) H1299/DDP cells when exposed to cisplatin following transfection with miR-133b mimic or miR-NC. (E) Western blot detection of Bcl-2, Bax and survivin protein expression following cisplatin treatment in the blank, miR-NC and miR-133b mimic groups. Data are the mean \pm standard deviation of three separate experiments. ${ }^{*} \mathrm{P}<0.05$ as indicated. miR, microRNA; NSCLC, non-small cell lung cancer; CCK, Cell Counting kit; IC $_{50}$, half maximal inhibitory concentration A549/DDP, cisplatin-resistant A549; H1299/DDP, cisplatin-resistant H1299; NC, negative control; Bcl-2, B-cell lymphoma 2; Bax, Bcl-2-associated X protein.

mimic into 293T cells. When the 293T cells were transfected with the WT GSTP1 3'-UTR, co-transfection with miR-133b mimic significantly inhibited luciferase activity $(\mathrm{P}<0.05$; Fig. 5B). By contrast, the effects of the miR-133b mimic were eliminated in 293T cells transfected with the mutant type GSTP1 3'-UTR. These results suggest that miR-133b binds directly to putative GSTP1 3'-UTR regions, as predicted. Furthermore, the protein expression and mRNA levels of GSTP1 in the cells following transfection with miR-133b mimic were examined. Western blotting and RT-qPCR revealed that the restoration of miR-133b markedly decreased GSTP1 protein (Fig. 5C) but not mRNA expression in A549/DDP and H1299/DDP cells 

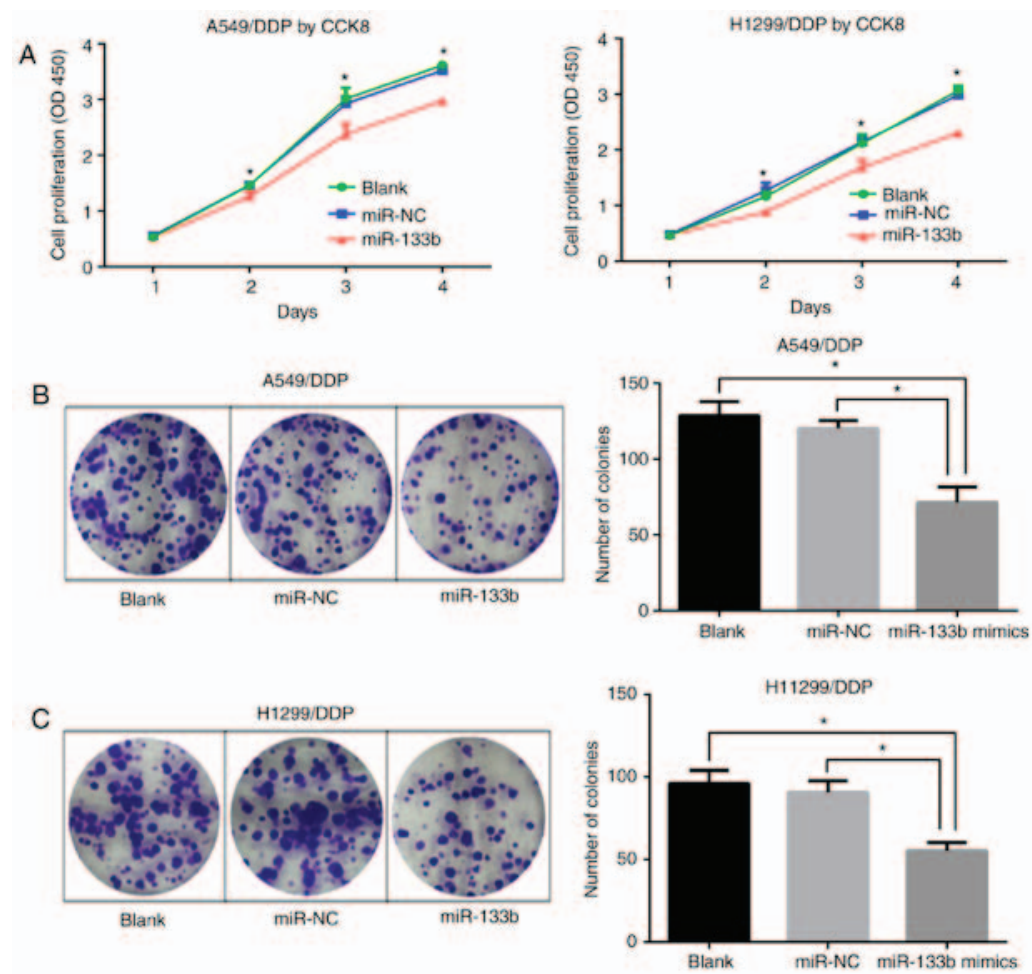

Figure 3. miR-133b inhibits the proliferation and clonogenesis of A549/DDP and H1299/DDP cells. (A) Overexpression of miR-133b reduces the cell viability of A549/DDP and H1299/DDP cells as determined using the Cell Counting kit-8 assay. Cell clone formation in the blank, miR-NC and miR-133b mimic groups of (B) A549/DDP and (C) H1299/DDP cells. Data are the means \pm standard deviation of three separate experiments. " $\mathrm{P}<0.05$ for miR-133b mimic vs. the blank and miR-NC groups. miR, microRNA; A549/DDP, cisplatin-resistant A549; H1299/DDP, cisplatin-resistant H1299; NC, negative control; OD450, optical density at $450 \mathrm{~nm}$.
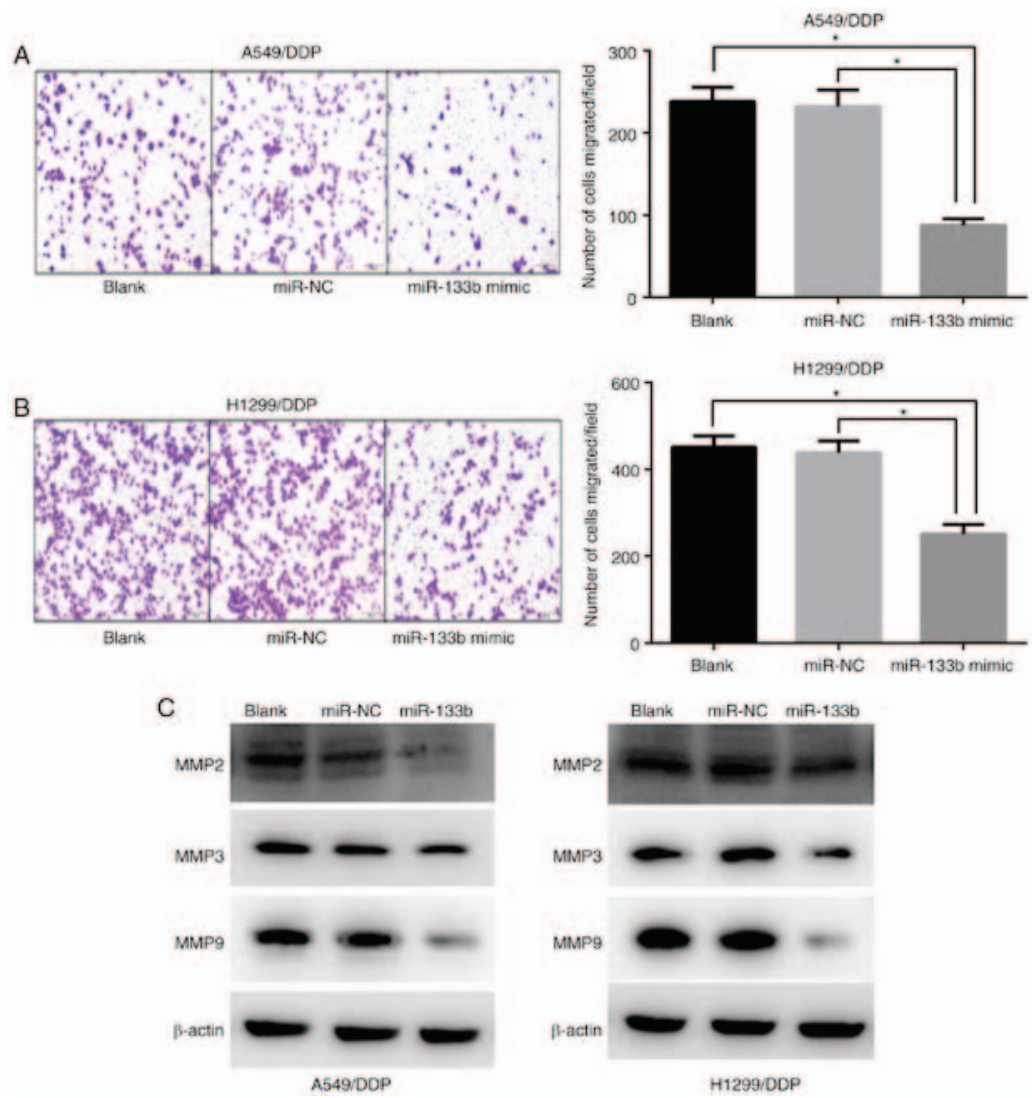

Figure 4. miR-133b attenuates the migration of A549/DDP and H1299/DDP cells. (A) Transwell migration assays for (A) A549/DDP cells and (B) H1299/DDP cells were conducted following transfection with miR-133b mimic or miR-NC. (C) Western blot detection of MMP-2, MMP-3 and MMP-9 protein expression in the blank, miR-NC and miR-133b mimic groups. Data are the mean \pm standard deviation of three separate experiments. "P $<0.05$ as indicated. miR, microRNA; A549/DDP, cisplatin-resistant A549; H1299/DDP, cisplatin-resistant H1299; NC, negative control; MMP, matrix metalloproteinase. 
A
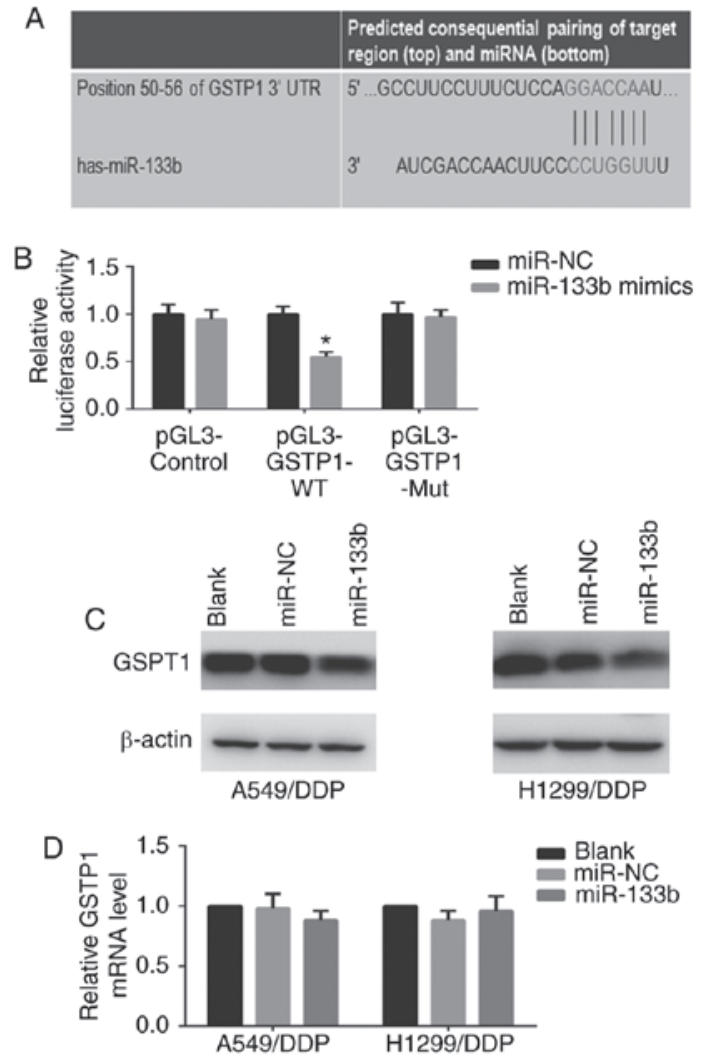

Figure 5. GSTP1 is a direct target of miR-133b. (A) Sequence alignment of miR-133b with the putative binding sites within the WT 3'-UTR of GSTP1. (B) Relative luciferase activity was analyzed in WT or Mut 3'-UTR reporter plasmids cotransfected with miR-133b mimic or miR-NC. (C) Western blot analysis of GSTP1 protein expression in A549/DDP and H1299/DDP cells transfected with miR-133b mimic or miR-NC. (D) Reverse transcription-quantitative polymerase chain reaction analysis of GSTP1 mRNA expression in A549/DDP and H1299/DDP cells transfected with miR-133b mimic or miR-NC. Data are the mean \pm standard deviation of three separate experiments. ${ }^{*} \mathrm{P}<0.05$ vs. the respective mimic-NC. GSTP1, glutathione-S-transferase P1; miR, microRNA; WT, wild type; Mut, mutant; UTR, untranslated region; NC, negative control; A549/DDP, cisplatin-resistant A549; H1299/DDP, cisplatin-resistant H1299.

(data not shown), indicating the potential post-transcriptional control of GSTP1 expression by miR-133b. Collectively, these findings confirm the existence of an inverse correlation between miR-133b and GSTP1 expression.

GSTP1 is critical in the miR-133b-mediated effects on chemosensitivity to cisplatin, cell proliferation and migration. The involvement of GSTP1 in the chemosensitivity to cisplatin, cell proliferation and migration of NSCLC cells following the regulation of miR-133b expression was investigated. The GSTP1 siRNA or NC siRNA was transfected into the A549/DDP and H1299/DDP cells, and the transfected cells were analyzed. The knockdown of GSTP1 significantly sensitized lung cancer cells to the induction of apoptosis by cisplatin (Fig. 6A and B) and attenuated the proliferation and migratory capabilities of the cells compared with those in the blank and NC groups (Fig. 6C and D). Furthermore, the western blotting results for GSTP1 silencing were comparable with those for miR-133b overexpression in the A549/DDP and H1299/DDP cells, and revealed the downregulation of Bcl-2 and survivin protein expression and the upregulation of Bax, accompanied by a marked reduction in MMP expression compared with the respective values in blank and NC groups (Fig. 6E). These results indicate that GSTP1 serves a critical role in the miR-133b-mediated effects on chemosensitivity to cisplatin, cell proliferation and migration.

\section{Discussion}

In the present study, it was revealed that miR-133b partly reverses cisplatin-resistance and inhibits the growth and migration of cisplatin-resistant NSCLC cells. To the best of our knowledge, this is the first study demonstrating that miR-133b confers cisplatin sensitivity and inhibits cell proliferation and migration by targeting GSTP1 in cisplatin-resistant lung cancer cells.

miRNAs have been indicated to regulate multi-drug resistance to chemotherapeutic reagents (19). With regard to miR-133b, Chen et al (20) reported that miRNA-133b increased the sensitivity of ovarian cancer cells to chemotherapeutic drugs, including cisplatin and paclitaxel. Zhou et al (21) demonstrated that combinational treatment with microRNA-133b and cetuximab exhibited increased inhibitory effects on the growth and invasion of colorectal cancer cells compared with either agent used alone. However, until now, no study has focused on the association between miR-133b and cisplatin resistance in cisplatin-resistant lung cancer. In the present study, it was demonstrated that miR-133b was downregulated in A549/DDP and H1299/DDP cells compared with the respective parental cells. Additionally, A549/DDP cells displayed stronger responses to cisplatin following miR-133b mimic transfection, as did H1299/DDP cells, indicating that miR-133b is a modulator of cisplatin resistance in NSCLC.

Although the overwhelming majority of studies support the function of miR-133b as a tumor suppressor in various cancers, Qin et al (22) suggested that miR-133b stimulates the progression of cervical carcinoma, indicating that miR-133b may have disparate effects in distinct cell environments. Notably, miRNAs are known to play multiple roles in different tissues depending on the expression of their target genes, as well as other tissue-specific modulating and regulatory factors $(23,24)$. In the present study, the ectopic expression of miR-133b repressed the tumorigenesis and metastasis of cisplatin-resistant NSCLC cells by attenuating their proliferation and migratory capabilities, which suggests that miR-133b acts as a tumor suppressor in lung cancer.

miRNAs are known to regulate the expression of multiple target genes and affect a variety of cellular pathways. Nevertheless, the particular pathways affected by miR-133b and the underlying mechanisms remain unclear. Using TargetScan, an in silico prediction tool, GSTP1 was identified as a target gene of miR-133b. GSTP1 belongs to a family of enzymes fulfilling protective and detoxifying functions in cells $(25,26)$. In addition, GSTP1 is frequently overexpressed in solid tumors and has been implicated in resistance against chemotherapy agents (27-29). Previously, Sau et al (30) reported that targeting GSTP1 leads to apoptosis in cisplatin-sensitive and -resistant human osteosarcoma cell lines. Sawers et al (31) found that GSTP1 directly influences the chemosensitivity of ovarian tumor cell lines to platinum drugs. In the present study, GSTP1 was validated as a direct target gene for miR-133b and GSTP1 knockdown was observed to increase the sensitivity of cisplatin-resistant NSCLC cells to cisplatin. Furthermore, 

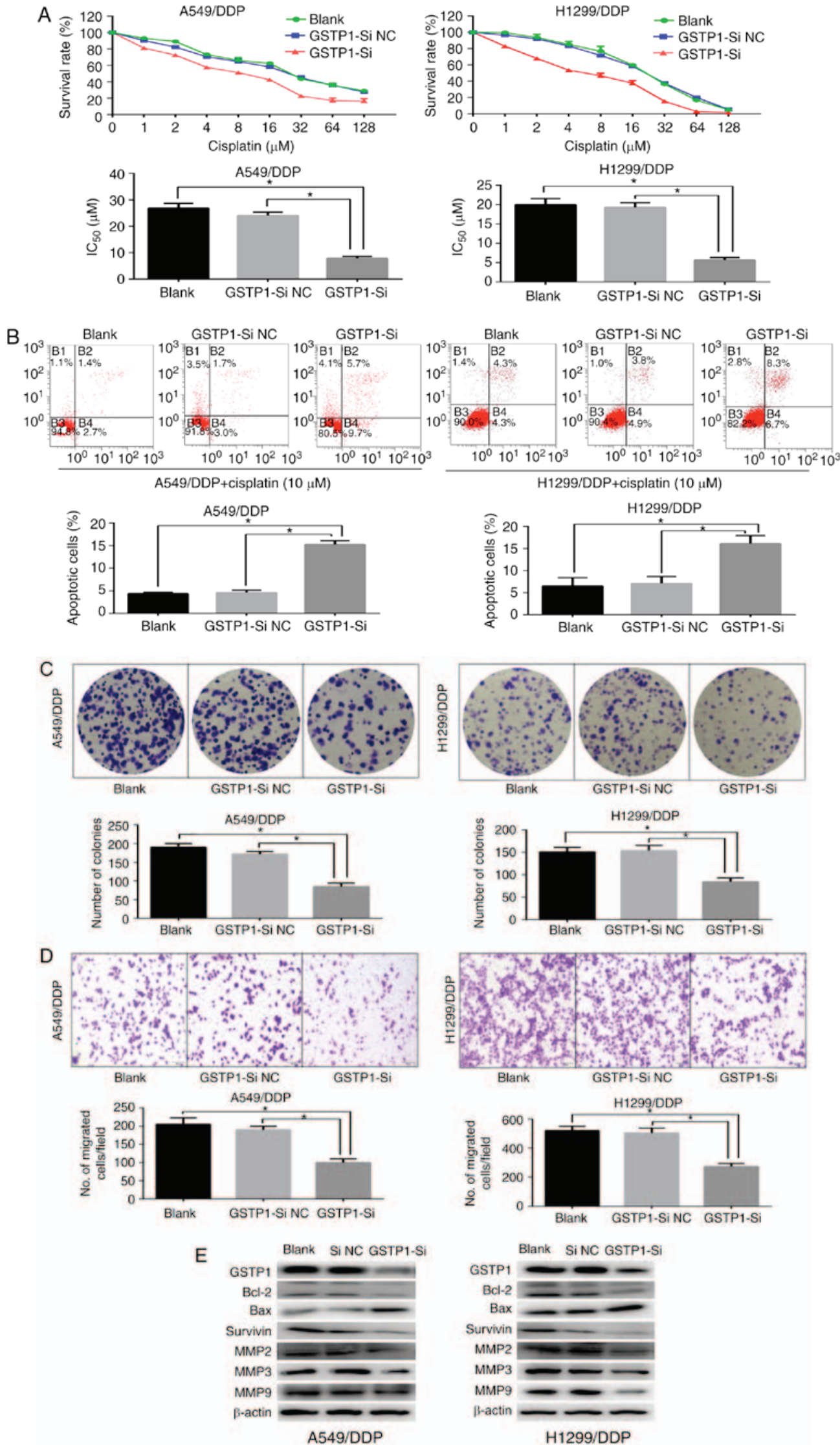

Figure 6. GSTP1 has a critical role in the miR-133b-mediated chemosensitivity of cisplatin-resistant NSCLC cells to cisplatin, cell proliferation and migration. (A) Cell Counting kit-8 and (B) flow cytometric assays demonstrate that the knockdown of GSTP1 sensitizes lung cancer cells to cisplatin-induced apoptosis. (C) Cell clone formation and (D) Transwell migration assays indicate that the knockdown of GSTP1 hampers the proliferation and migratory capabilities of the cells. (E) Western blot analysis of changes in the expression of apoptotic and migratory protein following GSTP1 knockdown. Data are the mean \pm standard deviation of three separate experiments. "P<0.05 as indicated. GSTP1, glutathione-S-transferase P1; miR, microRNA; NSCLC, non-small cell lung cancer; A549/DDP, cisplatin-resistant A549; H1299/DDP, cisplatin-resistant H1299; GSTP1-si, small interfering RNA against GSTP1; NC, negative control; Bcl-2, B-cell lymphoma 2; Bax, Bcl-2-associated X protein; MMP, matrix metalloproteinase. 
although GSTP1 protects tumor cells from apoptosis, little is known about its impact on tumor invasion and migration (32). The present study provides evidence that the repression of GSTP1 reduces the migration of cisplatin-resistant NSCLC cells, and suggests that GSTP1 may be a promising therapeutic target for the inhibition of tumor metastasis.

There are certain limitations to the present study. For example, the signaling pathways by which GSTP1 mediates its effects have yet to be further clarified. Also, validation in clinical samples or animal models is necessary to corroborate the results. However, the findings of the present study provide the insights that miR-133b partly reverses cisplatin resistance and its overexpression contributes to the suppression of the malignant growth and aggressiveness of cisplatin-resistant lung cancer cells by targeting GSTP1. This could be exploited as a novel therapeutic strategy to overcome cisplatin resistance.

\section{Acknowledgements}

The present study was supported by the National Natural Science Foundation of China (grant no. 81401896) and the Shanghai Science and Technology Committee (grant no. 124119a6200).

\section{Competing interests}

The authors declare that they have no competing interests.

\section{References}

1. Travis WD, Travis LB and Devesa SS: Lung cancer. Cancer 75 (1 Suppl): S191-S202, 2015.

2. Torre LA, Bray F, Siegel RL, Ferlay J, Lortet-Tieulent J and Jemal A: Global cancer statistics, 2012. CA Cancer J Clin 65: 87-108, 2015.

3. MitsudomiT,MoritaS, Yatabe Y,NegoroS,OkamotoI,Tsurutani J, Seto T, Satouchi M, Tada H, Hirashima T, et al: Gefitinib versus cisplatin plus docetaxel in patients with non-small-cell lung cancer harbouring mutations of the epidermal growth factor receptor (WJTOG3405): An open label, randomised phase 3 trial. Lancet Oncol 11: 121-128, 2010.

4. Oliver TG, Mercer KL, Sayles LC, Burke JR, Mendus D, Lovejoy KS, Cheng MH, Subramanian A, Mu D, Powers S, et al: Chronic cisplatin treatment promotes enhanced damage repair and tumor progression in a mouse model of lung cancer. Genes Dev 24: 837-52, 2010.

5. Ardizzoni A, Boni L, Tiseo M, Fossella FV, Schiller JH, Paesmans M, Radosavljevic D, Paccagnella A, Zatloukal P, Mazzanti P, et al: Cisplatin-versus carboplatin-based chemotherapy in first-line treatment of advanced non-small-cell lung cancer: An individual patient data meta-analysis. J Natl Cancer Inst 99: 847-857, 2007.

6. Tan XL, Moyer AM, Fridley BL, Schaid DJ, Niu N, Batzler AJ, Jenkins GD, Abo RP, Li L, Cunningham JM, et al: Genetic variation predicting cisplatin cytotoxicity associated with overall survival in lung cancer patients receiving platinum-based chemotherapy. Clin Cancer Res 17: 5801-5811, 2010.

7. Cortés-Sempere M, de Miguel MP, Pernía O, Rodriguez C, de Castro Carpeño J, Nistal M, Conde E, López-Ríos F, Belda-Iniesta C, Perona R and Ibanez de Caceres I: IGFBP-3 methylation-derived deficiency mediates the resistance to cisplatin through the activation of the IGFIR/Akt pathway in non-small cell lung cancer. Oncogene 32: 1274-1283, 2013.

8. Orellana EA and Kasinski AL: MicroRNAs in cancer: A historical perspective on the path from discovery to therapy. Cancers (Basel) 7: 1388-1405, 2015.

9. Bartel DP: MicroRNAs: Genomics, biogenesis, mechanism, and function. Cell 116: 281-297, 2004.

10. Chitwood DH and Timmermans MC: Small RNAs are on the move. Nature 467: 415-419, 2010.
11. Zhai $\mathrm{H}$ and Ju J: Implications of microRNAs in colorectal cancer development, diagnosis, prognosis, and therapeutics. Front Genet 2: 00078, 2011

12. Zhao JJ, Chu ZB, Hu Y, Lin J, Wang Z, Jiang M, Chen M, Wang X, Kang Y,ZhouY,etal:Targeting themiR-221-222/PUMA/BAK/BAX pathway abrogates dexamethasone resistance in multiple myeloma. Cancer Res 75: 4384-4397, 2015.

13. Ma J, Fang B, Zeng F, Ma C, Pang H, Cheng L, Shi Y, Wang H, Yin B, Xia J, et al: Down-regulation of miR-223 reverses epithelial-mesenchymal transition in gemcitabine-resistant pancreatic cancer cells. Oncotarget 6: 1740-1749, 2015.

14. Zhao Y, Huang J, Zhang L, Qu Y, Li J, Yu B, Yan M, Yu Y, Liu B and Zhu Z: MiR-133b is frequently decreased in gastric cancer and its overexpression reduces the metastatic potential of gastric cancer cells. BMC Cancer 14: 34, 2014.

15. Tao J, Wu D, Xu B, Qian W, Li P, Lu Q, Yin C and Zhang W: microRNA-133 inhibits cell proliferation, migration and invasion in prostate cancer cells by targeting the epidermal growth factor receptor. Oncol Rep 27: 1967-1975, 2012.

16. AkçakayaP,Ekelund S,Kolosenko I, Caramuta S, Ozata DM,XieH, Lindforss U, Olivecrona H and Lui WO: miR-185 and miR-133b deregulation is associated with overall survival and metastasis in colorectal cancer. Int J Oncol 9: 311-318, 2011.

17. Liu L, Shao X, Gao W, Zhang Z, Liu P, Wang R, Huang P, Yin Y and Shu Y: MicroRNA-133b inhibits the growth of non-small-cell lung cancer by targeting the epidermal growth factor receptor. FEBS J 279: 3800-3812, 2012.

18. Livak KJ and Schmittgen TD: Analysis of relative gene expression data using real-time quantitative PCR and the 2(-Delta Delta C(T)) method. Methods 25: 402-408, 2001.

19. MacDonagh L, Gray SG, Finn SP, Cuffe S, O'Byrne KJ and Barr MP: The emerging role of microRNAs in resistance to lung cancer treatments. Cancer Treat Rev 41: 160-169, 2015.

20. Chen S, Jiao JW, Sun KX, Zong ZH and Zhao Y: MicroRNA-133b targets glutathione $S$-transferase $\pi$ expression to increase ovarian cancer cell sensitivity to chemotherapy drugs. Drug Des Dev Ther 9: 5225-5235, 2015.

21. Zhou J, Lv L, Lin C, Hu G, Guo Y, Wu M, Tian B and Li X: Combinational treatment with microRNA 133b and cetuximab has increased inhibitory effects on the growth and invasion of colorectal cancer cells by regulating EGFR. Mol Med Rep 12: 5407-5414, 2015.

22. Qin W, Dong P, Ma C, Mitchelson K, Deng T, Zhang L, Sun Y, Feng X, Ding Y, Lu X, et al: MicroRNA-133b is a key promoter of cervical carcinoma development through the activation of the ERK and AKT1 pathways. Oncogene 31: 4067-4075, 2012.

23. Mendell JT: MiRiad roles for the miR-17-92 cluster in development and disease. Cell 133: 217-222, 2008.

24. Garzon R, Calin GA and Croce CM: MicroRNAs in cancer. Annu Rev Med 60: 167-179, 2009.

25. Singh S: Cytoprotective and regulatory functions of glutathione $\mathrm{S}$-transferases in cancer cell proliferation and cell death. Cancer Chemother Pharmacol 75: 1-15, 2015.

26. Schnekenburger M, Karius T and Diederich M: Regulation of epigenetic traits of the glutathione S-transferase P1 gene: From detoxification toward cancer prevention and diagnosis. Front Pharmacol 5: 170, 2014.

27. Louie SM, Grossman EA, Crawford LA, Ding L, Camarda R, Huffman TR, Miyamoto DK, Goga A, Weerapana E and Nomura DK: GSTP1 is a driver of triple-negative breast cancer cell metabolism and pathogenicity. Cell Chem Biol 23: 567-578, 2016.

28. Townsend DM and Tew KD: The role of glutathione-S-transferase in anti-cancer drug resistance. Oncogene 22: 7369-7375, 2003.

29. Depeille P, Cuq P, Passagne I, Evrard A and Vian L: Combined effects of GSTP1 and MRP1 in melanoma drug resistance. Br J Cancer 93: 216-223, 2005.

30. Sau A, Filomeni G, Pezzola S, D'Aguanno S, Tregno FP, Urbani A, Serra M, Pasello M, Picci P, Federici G and Caccuri AM: Targeting GSTP1-1 induces JNK activation and leads to apoptosis in cisplatin-sensitive and -resistant human osteosarcoma cell lines. Mol Biosyst 8: 994-1006, 2011.

31. Sawers L,Ferguson MJ,Ihrig BR, YoungHC,ChakravartyP,WolfCR and Smith G: Glutathione S-transferase P1 (GSTP1) directly influences platinum drug chemosensitivity in ovarian tumour cell lines. Br J Cancer 111: 1150-1158, 2014.

32. Holley SL, Fryer AA, Haycock JW, Grubb SE, Strange RC and Hoban PR: Differential effects of glutathione S-transferase pi (GSTP1) haplotypes on cell proliferation and apoptosis. Carcinogenesis 28: 2268-2273, 2007.

This work is licensed under a Creative Commons Attribution-NonCommercial-NoDerivatives 4.0 International (CC BY-NC-ND 4.0) License. 